\title{
Glycoprotein M6A (GPM6A) is over-expressed in brain metastatic breast cancer.
}

Shahan Mamoor, $\mathrm{MS}^{1}$

1shahanmamoor@gmail.com

East Islip, NY USA

One study reported the incidence of central nervous system metastases in breast cancer patients treated with trastuzumab as $34 \%{ }^{1}$. We mined published microarray data ${ }^{2,3}$ to discover genes associated with brain metastasis in breast cancer. We identified significant differential expression of glycoprotein M6A, encoded by GPM6A ${ }^{4,5}$, in the brain metastases of patients with metastatic breast cancer. GPM6A may be relevant to the biology underlying colonization of the brain with metastatic breast cancer clones.

Keyword: breast cancer, metastasis, brain metastasis, central nervous system metastasis, GPM6A, systems biology of breast cancer, targeted therapeutics in breast cancer. 
One study reported a 34\% incidence of central nervous system metastases in patients with breast cancer treated with the monoclonal antibody trastuzumab (Herceptin) ${ }^{1}$. Treatment options for patients with brain metastatic breast cancer are limited ${ }^{6}$. We mined published microarray datasets ${ }^{2,3}$ to understand in an unbiased and systems-level fashion genes whose expression most significantly distinguished brain metastases from primary tumors of the breast, the cells from which these metastatic clones are presumably derived. We found significant differential expression of GPM6A in the brain metastases of patients with brain metastatic breast cancer.

\section{Methods}

We utilized microarray datasets GSE $125989^{2}$ and GSE100534³ for this differential gene expression analysis of brain metastatic breast cancer in conjunction with GEO2R. GSE100534 was generated using Affymetrix Human Gene 1.0 ST Array technology with $n=16$ primary tumors from patients with breast cancer and $n=3$ brain metastases from patients with brain metastatic breast cancer. GSE43837 was generated using Affymetrix Human X3P Array technology with $n=19$ primary breast tumors and $n=19$ brain metastases from patients with breast cancer, analysis performed using platform GPL1352. The Benjamini and Hochberg method of $p$-value adjustment was used for ranking of differential expression but raw $p$-values were used to assess statistical significance of global differential expression. Log-transformation of data was auto-detected, and the NCBI generated category of platform annotation was used. A statistical test was performed to evaluate whether GPM6A gene expression was significantly between primary breast tumors and brain metastases in humans with breast cancer using a two-tailed, unpaired t-test with Welch's correction. We used PRISM for all statistical analyses of differential gene expression in human breast cancer (Version 8.4.0)(455).

\section{Results}

We mined published microarray data ${ }^{2,3}$ to discover genes associated with brain metastasis in breast cancer.

\section{GPM6A is differentially expressed in brain metastatic breast cancer.}

We identified glycoprotein M6A, encoded by GPM6A, as among the genes whose expression changes most significantly when comparing brain metastases to primary tumors of the breast in patients with brain metastatic breast cancer ${ }^{2}$ (Table 1). When sorting each of the genes expressed in brain metastases based on significance of change as compared to primary tumors of the breast, GPM6A ranked 176 out of 22277 total transcripts (Table 1), equating to $99.2 \%$ differential expression. Differential expression of GPM6A in brain metastases in brain metastatic breast cancer was statistically significant (Table $1 ; p=1.18 \mathrm{E}-03)$.

We queried a second microarray dataset ${ }^{3}$ to validate transcriptome-wide differential expression of GPM6A in brain metastatic breast cancer. We again found that GPM6A was among the genes whose expression changed most significantly when comparing metastases of the brain to primary tumors of the breast in patients with breast cancer (Table 2). When sorting each of the genes expressed in brain metastases based on significance of change as compared to primary tumors of the breast, GPM6A ranked 4772 out of 33297 total transcripts (Table 2), equating to $85.7 \%$ differential expression. Differential expression of GPM6A in brain metastases in brain metastatic breast cancer was statistically significant (Table $2 ; p=1.45 \mathrm{E}-02)$. 
GPM6A is expressed at significantly higher levels in brain metastases as compared to primary tumors of the breast in brain metastatic breast cancer.

We obtained exact mRNA expression values for GPM6A from brain metastases and primary tumors of the breast to evaluate magnitude and direction of change in GPM6A expression in brain metastases in patients with breast cancer. GPM6A was expressed at higher levels in brain metastases in patients with breast cancer and this difference was statistically significant (Figure $1 ; p=0.0018$ ). We calculated a mean fold change of $1.61 \pm 0.55$ in GPM6A expression when comparing brain metastatic tissues to primary tumors of the breast (Table 1).

Thus, by mining published microarray data ${ }^{2,3}$, we found that GPM6A was among the genes, globally, whose expression was most different when comparing primary tumors of the breast to brain metastases in patients with breast cancer; GPM6A mRNA was present at significantly higher quantities in brain metastases as compared to primary tumors of the breast in patients with metastatic breast cancer.

\section{Discussion}

GPM6A spans 274 amino acids in length and is a membrane glycoprotein with expression originally reported as specific to the brain 4,5 . Structural features of GPM6A include four transmembrane domains, two extracellular loops, and a small intracellular loop ${ }^{7}$. In human embryonic stem cells, GPM6A ectopic expression results in neuroectoderm-associated genes, including Pax2 and Sox2, as well increased numbers of hES cell-derived neural stem cells ${ }^{8}$. More specifically, expression of M6A can be observed in the hippocampus; in hippocampal neurons, M6A ectopic expression results in the neurite formation and an increased filopodia density 9 . In hippocampal neurons from the rat, localization of GPM6A can be observed in lipid rafts with ganglioside GM-1, "floating" with flotillin-1 in a detergentinsoluble fraction ${ }^{10}$. In Graffi murine leukemia virus induced-primary B-cell malignancies, overexpression of GPM6A was found, with ectopic expression of GPM6A in NIH 3T3 cells resulting in increased cell proliferation and anchorage-independent cell growth in vitro ${ }^{11}$. Thus, GPM6A is a membrane glycoprotein expressed through the brain, localized to lipid rafts and possessing ability to promote generation of neurites; GPM6A can support the growth of neural stem cells and gene expression associated with the neuroectoderm, and GPM6A expression is up-regulated in virally-induced B-cell cancer in mice where it can support cancer cell proliferation.

We reported here that the brain-expressed membrane glycoprotein GPM6A was among the genes whose expression was most quantitatively different in metastases to the brain when compared to primary tumors of the breast in patients with metastatic breast cancer. GPM6A may be of relevance to the biology underlying any number of steps important for metastasis to the brain: exit from the breast, traversing the vasculature and the blood-brain barrier, and colonization of the brain. 


\section{References}

1. Bendell, J.C., Domchek, S.M., Burstein, H.J., Harris, L., Younger, J., Kuter, I., Bunnell, C., Rue, M., Gelman, R. and Winer, E., 2003. Central nervous system metastases in women who receive trastuzumab-based therapy for metastatic breast carcinoma. Cancer, 97(12), pp.2972-2977.

2. Iwamoto, T., Niikura, N., Ogiya, R., Yasojima, H., Watanabe, K.I., Kanbayashi, C., Tsuneizumi, M., Matsui, A., Fujisawa, T., Iwasa, T. and Shien, T., 2019. Distinct gene expression profiles between primary breast cancers and brain metastases from pair-matched samples. Scientific reports, $9(1)$, pp. $1-8$.

3. Schulten, H.J., Bangash, M., Karim, S., Dallol, A., Hussein, D., Merdad, A., Al-Thoubaity, F.K., AlMaghrabi, J., Jamal, A., Al-Ghamdi, F. and Choudhry, H., 2017. Comprehensive molecular biomarker identification in breast cancer brain metastases. Journal of translational medicine, 15(1), p.269.

4. Shimizu, F., Watanabe, T.K., Fujiwara, T., Takahashi, E., Nakamura, Y. and Maekawa, H., 1996. Isolation and mapping of the human glycoprotein M6 gene (GPM6A) to 4q33 $\rightarrow$ q34. Cytogenetic and Genome Research, 74(1-2), pp.138-139.

5. Yan, Y., Lagenaur, C. and Narayanan, V., 1993. Molecular cloning of M6: identification of a PLP/ DM20 gene family. Neuron, 11(3), pp.423-431.

6. Robson, M., Im, S.A., Senkus, E., Xu, B., Domchek, S.M., Masuda, N., Delaloge, S., Li, W., Tung, N., Armstrong, A. and Wu, W., 2017. Olaparib for metastatic breast cancer in patients with a germline BRCA mutation. New England Journal of Medicine, 377(6), pp.523-533.

7. Rosas, N.M., Alvarez Juliá, A., Alzuri, S.E., Frasch, A.C. and Fuchsova, B., 2018. Alanine scanning mutagenesis of the C-terminal cytosolic end of Gpm6a identifies key residues essential for the formation of filopodia. Frontiers in molecular neuroscience, 11, p.314.

8. Michibata, H., Okuno, T., Konishi, N., Kyono, K., Wakimoto, K., Aoki, K., Kondo, Y., Takata, K., Kitamura, Y. and Taniguchi, T., 2009. Human GPM6A is associated with differentiation and neuronal migration of neurons derived from human embryonic stem cells. Stem cells and development, 18(4), pp.629-640.

9. Alfonso, J., Fernández, M.E., Cooper, B., Flugge, G. and Frasch, A.C., 2005. The stress-regulated protein M6a is a key modulator for neurite outgrowth and filopodium/spine formation. Proceedings of the National Academy of Sciences, 102(47), pp.17196-17201.

10.Scorticati, C., Formoso, K. and Frasch, A.C., 2011. Neuronal glycoprotein M6a induces filopodia formation via association with cholesterol-rich lipid rafts. Journal of neurochemistry, 119(3), pp. 521-531.

11.Charfi, C., Edouard, E. and Rassart, E., 2014. Identification of GPM6A and GPM6B as potential new human lymphoid leukemia-associated oncogenes. Cellular Oncology, 37(3), pp.179-191. 
PAGE 6 
Figure 1: GPM6A is expressed at significantly higher levels in brain metastases as compared to
primary tumors of the breast in patients with brain metastatic breast cancer.

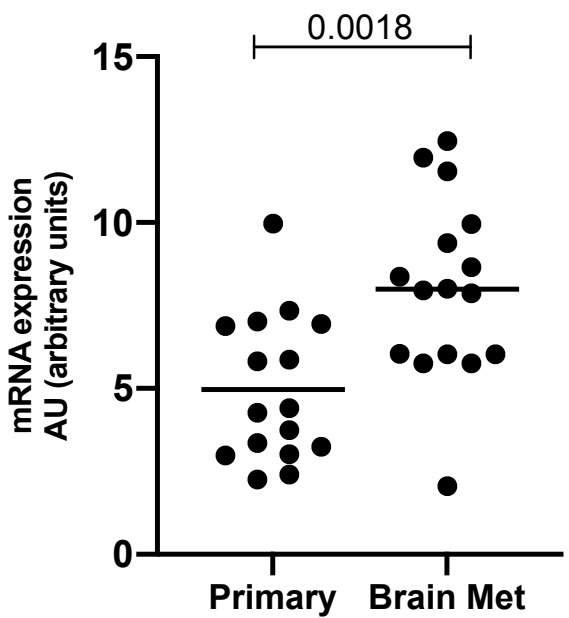

The mRNA expression level of GPM6A in primary tumors of the breast (left) and in the brain metastases of brain metastatic breast cancer patients (right) is graphically represented here with mean GPM6A levels marked and the result of a statistical test evaluating difference in mRNA expression level between brain metastases and primary tumors of the breast, a $p$-value, listed above. 